\title{
Clerodendrum inerme Leaf Extract Alleviates Animal Behaviors, Hyperlocomotion, and Prepulse Inhibition Disruptions, Mimicking Tourette Syndrome and Schizophrenia
}

\author{
Hon-Lie Chen, ${ }^{1}$ Hsin-Jung Lee, ${ }^{2}$ Wei-Jan Huang, ${ }^{3}$ Jui-Feng Chou, ${ }^{1}$ Pi-Chuan Fan, ${ }^{1,4}$ \\ Jung-Chieh Du, ${ }^{1,5}$ Yuan-Ling Ku, ${ }^{6}$ and Lih-Chu Chiou ${ }^{1,2,7}$ \\ ${ }^{1}$ Graduate Institute of Pharmacology, College of Medicine, National Taiwan University, No. 1, Jen-Ai Road, Section 1, \\ Taipei 100, Taiwan \\ ${ }^{2}$ Department of Pharmacology, College of Medicine, National Taiwan University, No. 1, Jen-Ai Road, Section 1, \\ Taipei 100, Taiwan \\ ${ }^{3}$ Graduate Institute of Pharmacognosy, Taipei Medical University, No. 250, Wu-Hsing Street, Taipei 110, Taiwan \\ ${ }^{4}$ Department of Pediatrics, College of Medicine, National Taiwan University, No. 1, Jen-Ai Road, Section 1, Taipei 100, Taiwan \\ ${ }^{5}$ Department of Pediatrics, Taipei City Hospital, Zhongxiao Branch, No. 87, Tongde Road, Taipei 115, Taiwan \\ ${ }^{6}$ Medical and Pharmaceutical Industry Technology and Development Center, 7F, No. 9, Wuquan Road, Wugu Dist., \\ New Taipei 248, Taiwan \\ ${ }^{7}$ Graduate Institute of Brain and Mind Sciences, College of Medicine, National Taiwan University, No. 1, Jen-Ai Road, \\ Section 1, Taipei 100, Taiwan
}

Correspondence should be addressed to Lih-Chu Chiou, lcchiou@ntu.edu.tw

Received 23 February 2012; Accepted 1 June 2012

Academic Editor: Ching Liang Hsieh

Copyright () 2012 Hon-Lie Chen et al. This is an open access article distributed under the Creative Commons Attribution License, which permits unrestricted use, distribution, and reproduction in any medium, provided the original work is properly cited.

\begin{abstract}
Previously, we found a patient with intractable motor tic disorder, a spectrum of Tourette syndrome (TS), responsive to the ground leaf juice of Clerodendrum inerme $(C I)$. Here, we examined the effect of the ethanol extract of $C I$ leaves $(C I$ extract) on animal behaviors mimicking TS, hyperlocomotion, and sensorimotor gating deficit. The latter is also observed in schizophrenic patients and can be reflected by a disruption of prepulse inhibition of acoustic startle response (PPI) in animal models induced by methamphetamine and NMDA channel blockers (ketamine or MK-801), based on hyperdopaminergic and hypoglutamatergic hypotheses, respectively. $C I$ extract $(10-300 \mathrm{mg} / \mathrm{kg}$, i.p.) dose-dependently inhibited hyperlocomotion induced by methamphetamine $(2 \mathrm{mg} / \mathrm{kg}$, i.p. ) and PPI disruptions induced by methamphetamine, ketamine ( $30 \mathrm{mg} / \mathrm{kg}$, i.p.), and MK- 801 $(0.3 \mathrm{mg} / \mathrm{kg}$, i.p. $)$ but did not affect spontaneous locomotor activity, rotarod performance, and grip force. These results suggest that $C I$ extract can relieve hyperlocomotion and improve sensorimotor gating deficit, supporting the therapeutic potential of CI for TS and schizophrenia.
\end{abstract}

\section{Introduction}

In previous case study, we found that a 13 year-old girl who had suffered from intractable chronic motor tic disorders for more than 6 years responded dramatically to the grounded leaf juice of a local herb, Clerodendrum inerme (L.) Gaertn $(C I)$. Her tics subsided 1 hour after taking this leaf juice. After taking the herb for 2 years, her motor tics were markedly reduced and her physical and laboratory examinations, including hemograms, liver and renal functions, blood gas, and electrolytes, were all normal [1].
The tic disorder is a neuropsychiatric disorder with a prevalence rate of $0.4-1 \%$ worldwide [2] and $5.5 \%$ in Taiwan [3], manifesting involuntary, sudden, rapid, repetitive, nonrhythmic, stereotyped movements (motor tics) or phonic tics [4]. Tourette syndrome (TS) is an idiopathic spectrum of tic disorders with multiple motor tics and at least one phonic tic lasting for at least one year and often comorbids with obsessive-compulsive disorder (OCD) and attention deficit hyperactivity disorder (ADHD) [5].

The pathogenesis of tic disorders or TS remains unclear although dopaminergic hyperreactivity in the basal ganglia 
and a deficit in cortico-thalamic-pallidostrial circuits have been proposed [4]. It is suggested that TS patients have overactive dopamine transporter system, resulting in reduced tonic dopamine release and upregulation of postsynaptic D2 receptors, while, upon stimulation, phasic dopamine release is increased, leading to hyperreactivity in motor responses. This hypothesis is based on the effectiveness of antipsychotic agents in clinical practices and the limited morphological studies in humans using postmortem brain tissues or clinical neuroimaging studies in patients $[6,7]$.

TS patients also manifest a sensorimotor gating deficit, which is believed to contribute to their premonitory urges [8] and can be reflected by an endophenotype deficit in prepulse inhibition of acoustic startle response (PPI) [9]. PPI is a neurophysiological phenomenon that the startle response to a stimulus (pulse) is inhibited if a weaker stimulus (prepulse) with the same source is preapplied within a short interval. The stimulus could be a sound, an airpuff, or a light. PPI is believed to be a processing protection in a living organism, serving as a preconscious regulator of attention, termed sensorimotor gating, to reduce the startle response that is harmful to the information professing. PPI can be assessed by the motor response in either humans (measuring the eyeblink response by electromyograph) or animals (measuring the startle jumping response) [10].

Not only in TS patients, PPI disruptions were also observed in patients with other neuropsychiatric disorders related to TS, such as $\operatorname{ADHD}[11,12]$, OCD [13], and schizophrenia [14]. ADHD and OCD are two common comorbidities of TS, and TS in childhood is a risk etiology of schizophrenia [15]. Especially in schizophrenic patients, PPI disruption is believed to be a typical endophenotype of cognitive function deficits, leading to hallucination due to a flood of sensory inputs [14]. Two hypotheses of schizophrenia, hyperdopaminergic and hypoglutamatergic, have been proposed and the respective animal models have been established. The models with animals treated with dopamine mimetic agents, such as methamphetamine, which facilitates dopamine release, or apomorphine, which is a dopamine receptor agonist, are based on the hyperdopaminergic hypothesis [16]. The models with animals treated with noncompetitive NMDA channel blockers, such as phencyclidine [17], ketamine, and MK-801 [16], are based on the hypoglutamatergic hypothesis. PPI disruptions can be induced in these animal models [18-21].

So far, no available animal models perfectly mimic TS or tic disorders. Nevertheless, based on the hyperdopaminergic hypothesis, we used a mouse model treated with methamphetamine that induced hyperlocomotion to examine if the leaf extract of $C I$ was effective in this model. In addition, we also examined if the $C I$ leaf extract was effective in rescuing PPI disruptions induced by methamphetamine, ketamine, and MK 801.

\section{Materials and Methods}

2.1. Plant Material. The leaves of CI were collected from mangrove marshes in the riverside of southern Taiwan in March 2005. The CI leave sample from the same batch collection was deposited as a voucher specimen (TMU27423) in the herbarium of College of Pharmacy, Taipei Medical University.

2.2. Preparation of the Ethanol Extract from CI Leaves. The air-dried CI leaves $(3.00 \mathrm{~kg})$ were ground and repeatedly extracted with $95 \%$ ethanol (10 L) three times, each for one week. The combined ethanol layers were evaporated under reduced pressure to give a residue $(246.26 \mathrm{~g})$. This ethanol extract of $C I$ leaves (CI Extract) was stored at $4^{\circ} \mathrm{C}$. When used in the subsequent animal experiments, it was dissolved in dimethylsulfoxide (DMSO).

2.3. Animal Experiments. All animal experiments were approved by the Institutional Animal Care and Use Committee of National Taiwan University, College of Medicine (NTUMC). Male ICR mice aged 6-9 weeks were housed (35 mice per cage) in the holding room of NTUMC Animal Center on a $12 \mathrm{~h} / 12 \mathrm{~h}$ reversed light schedule with free access to rodent chow and water. On the day of conducting behavioral tests, mice in their home cages were transferred to the behavioral room and acclimated for 1 hour before starting experiments. The testing chamber in each behavioral apparatus was cleaned with $70 \%$ alcohol after each mouse had completed a test session. All drugs were given by intraperitoneal (i.p.) injection with a volume of $0.1 \mathrm{~mL}$.

2.4. Locomotor Activity. The locomotor activity of the mouse was measured by its interruptions of infrared photobeams in a locomotor cage $(42 \mathrm{~cm} \times 42 \mathrm{~cm} \times 36 \mathrm{~cm})$ in the Photobeam Activity System (San Diego Instrument, San Diego, CA). After acclimation, the mouse was treated with CI extract or vehicle for $15 \mathrm{~min}$, followed by methamphetamine $(2 \mathrm{mg} / \mathrm{kg})$. Then, the mouse was gently placed in the locomotor cage and the horizontal and vertical interruptions were counted every $5 \mathrm{~min}$ for 2 hours. Total locomotor activity was the sum of interruptions within 2 hours.

2.5. Rotarod Test. The motor coordinating activity of the mouse was measured by its performance on a rotarod in the SDI ROTOR-ROD system (San Diego Instruments, San Diego, CA). The mouse was trained four times a day for 3 days until it could stay on the rotating drum at a rotating speed of $24 \mathrm{rpm}$ for at least $120 \mathrm{sec}$. Then, the mouse was subjected to the rotarod test at the rotating speed accelerating gradually from 0 to $30 \mathrm{rpm}$, and the latency to fall from the rotating drum was recorded. The cut-off time for the latency to fall was $300 \mathrm{sec}$. Animals were treated with $C I$ extract or vehicle for $15 \mathrm{~min}$ before receiving the test.

2.6. Grip Strength Test. Forelimb grip strength was measured by the grip force of forepaws of the mouse using the SDI Grip Strength System (San Diego Instrument, San Diego, CA). The grip strength was recorded by the maximum of three permissible readings (in grams). The test was conducted every $2 \mathrm{~min}$ for three times, and the averaged grip strength was recorded. Animals were treated with CI extract or vehicle for $15 \mathrm{~min}$ before the test. 
2.7. PPI Test. The PPI test was conducted with a PPI apparatus (SR-LAB, San Diego Instruments, San Diego, CA) consisting of a startle chamber equipped with various programming acoustic stimulations. After acclimation, the mouse was gently placed in the startle chamber for a $4 \mathrm{~min}$ acclimation period with a background noise of $65 \mathrm{~dB}$, which continued throughout the whole PPI test session. One PPI test session consisted of 4 types of startle trials, including the trial with the startle pulse $(115 \mathrm{~dB})$ alone (PULSEALONE; $115 \mathrm{~dB}, 20 \mathrm{~ms}$ ), two trials with the startle pulse paired with 71 and $77 \mathrm{~dB}$ prepulses, respectively (PREPULSE + PULSE; $71 \mathrm{~dB}+115 \mathrm{~dB}$ and $77 \mathrm{~dB}+115 \mathrm{~dB})$, and the trial without stimulus (NOSTIM; background $65 \mathrm{~dB}$ only). A test session started and ended, respectively, with four NOSTIM trials and four PULSEALONE trials. In between, each of the four types of trials was presented 14 times randomly, that is, 56 trials were given in a test session. The intertrial interval was given randomly from 5 to $20 \mathrm{~s}$. In the PREPULSE + PULSE trial, a 71 or $77 \mathrm{~dB}$ prepulse was given $120 \mathrm{~ms}$ before the $115 \mathrm{~dB}$ pulse. The magnitude of PPI (PPI\%) was determined, after summarizing the startle responses in PULSEALONE and PREPULSE + PULSE trials, according to the equation (PULSEALON - PREPULSE + PULSE)/PULSEALONE $\times$ $100 \%$. CI extract or vehicle was given to the animals for $15 \mathrm{~min}$, followed by methamphetamine $(2 \mathrm{mg} / \mathrm{kg})$, ketamine $(30 \mathrm{mg} / \mathrm{kg})$, or MK-801 $(0.3 \mathrm{mg} / \mathrm{kg})$. The PPI test was conducted $10 \mathrm{~min}$ after injection of methamphetamine or ketamine and 20 min after MK-801 injection.

2.8. Chemicals. Methamphetamine was purchased from Sigma-Aldrich (St. Louis, MO) and ketamine was purchased from Parke-Davis (Taoyuan, Taiwan) with the approval of Food and Drug Administration, Department of Health, Executive Yuan, Taiwan. MK-801, haloperidol, and clozapine were purchased from Sigma-Aldrich. CI extract, haloperidol, and clozapine were dissolved in DMSO. Methamphetamine, ketamine, and MK-801 were dissolved in normal saline.

2.9. Statistics. Data were expressed as the mean \pm S.E.M. Statistical comparisons among groups were analyzed by ANOVA with Tukey post hoc test, and differences between groups were analyzed by Student's $t$-test. Two-way ANOVA with Bonferroni's post hoc test was used to analyze differences in the time courses of locomotor activity among groups. Differences were considered significant if $P<0.05$.

\section{Results and Discussion}

\subsection{Effects of CI Extract on Motor Functions}

3.1.1. CI Extract Reduced Methamphetamine-Induced Hyperlocomotion at Doses without Affecting Motor Coordination and Muscle Power. In normal saline-treated mice, the spontaneous locomotor activity was markedly decreased within 10-15 min after they were placed in the locomotor cage due to acclimation (open squares in Figures 1(a) and 1(b)). In contrast, in mice treated with $2 \mathrm{mg} / \mathrm{kg}$ (i.p.) of methamphetamine, the locomotor activity was significantly increased. This hyperlocomotion, after a transient decrease, reached a peak at 20-30 min and declined gradually within 2 hours (open circles in Figure 1(a)). The total locomotor activity within 2 hours in methamphetamine-treated mice was significantly higher than that in the group without methamphetamine treatment $(22327 \pm 2309$ versus $4549 \pm$ 926 interruptions, $P<0.001$ ) (open bars in Figures 1(b) and $1(\mathrm{~d}))$.

Methamphetamine-induced hyperlocomotion was significantly decreased in the group pretreated with i.p. injection of CI Extract for $15 \mathrm{~min}$, as compared with the vehicle-pretreated group. This effect of $C I$ extract was dosedependent at tested doses ranging from 10 to $300 \mathrm{mg} / \mathrm{kg}$ (Figures 1(a) and 1(c)). On the other hand, in mice without treatment with methamphetamine, $C I$ extract did not alter spontaneous locomotor activity (Figures $1(\mathrm{~b})$ and $1(\mathrm{~d})$ ).

3.1.2. CI Extract Did Not Affect the Rotarod Performance and Grip Strength. We further examined the effects of $C I$ extract on other motor functions, including the motor coordination and muscle power, which were measured by the rotarod performance and grip strength, respectively. In the rotarod test, the performance in mice treated with $C I$ extract at the dose up to $300 \mathrm{mg} / \mathrm{kg}$ was not significantly different from that in vehicle-treated mice (Figure 2(a)). The grip strength, a measurement for muscle contraction power, was also unaffected by $C I$ extract at the dose up to $300 \mathrm{mg} / \mathrm{kg}$ (Figure 2(b)).

These results indicate that the ethanol extract of $C I$ leaves inhibited methamphetamine-induced hyperlocomotion at doses without affecting spontaneous locomotor activity or other motor functions, such as motor coordination and muscle power.

\subsection{Effect of CI Extract on PPI Disruptions}

3.2.1. Establishing a PPI Disruption Model Induced by Methamphetamine. In addition to tic attacks, TS patients also manifest a deficit in sensorimotor gating function, which can be measured by a disruption of PPI in humans or animal models. Increasing dopaminergic activity has been reported to disrupt PPI, and this disruption can be blocked by antipsychotic agents [22], which are also effective antitic drugs clinically. We, therefore, established a PPI disruption model in mice induced by i.p. injection of methamphetamine $(2 \mathrm{mg} / \mathrm{kg})$, which increases dopamine release. The startle response to a $115 \mathrm{~dB}$ acoustic stimulation in normal salinetreated mice was inhibited by a prepulse acoustic stimulation at $71 \mathrm{~dB}$ by $58.9 \pm 2.3 \%$, that is, the PPI magnitude in this $71-115 \mathrm{~dB}$ protocol is $58.9 \pm 2.3 \%$ (open bar in Figure $3(\mathrm{a})$ ). Increasing the prepulse acoustic stimulation to $77 \mathrm{~dB}$, the magnitude of PPI was increased to $65.6 \pm$ $3.1 \%$ (open slashed bar in Figure 3(a)). In animals treated with methamphetamine $(2 \mathrm{mg} / \mathrm{kg}$, i.p.), PPI was significantly reduced to $41.1 \pm 3.8 \%$ and $46.1 \pm 4.9 \%$, respectively, in response to $71-115 \mathrm{~dB}$ and $77-115 \mathrm{~dB}$ stimulations. In mice pretreated with the antipsychotic agent, either a typical one (haloperidol, $0.3 \mathrm{mg} / \mathrm{kg}$, i.p.) or an atypical one (clozapine, $1 \mathrm{mg} / \mathrm{kg}$, i.p.) for $15 \mathrm{~min}$, PPI was significantly restored to 


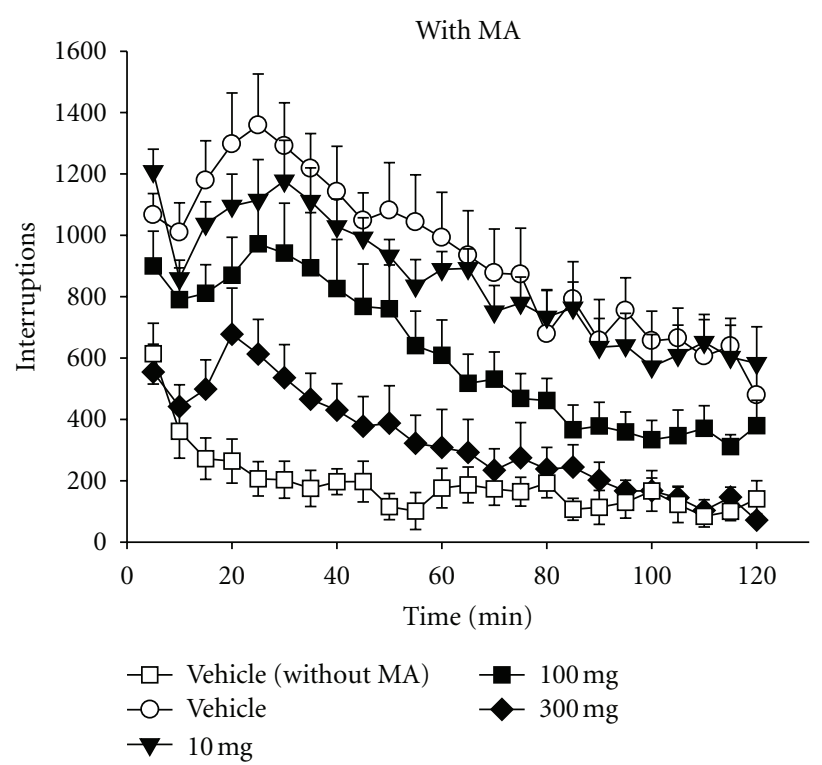

(a)

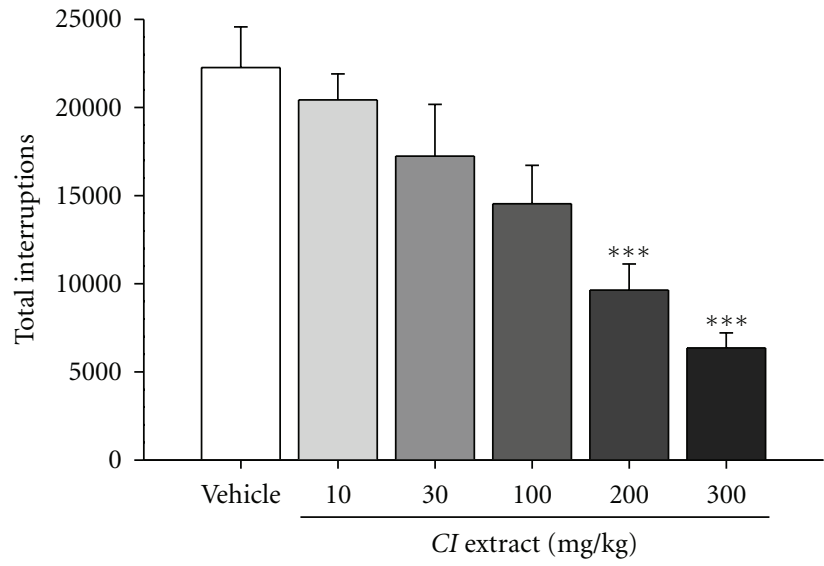

(c)

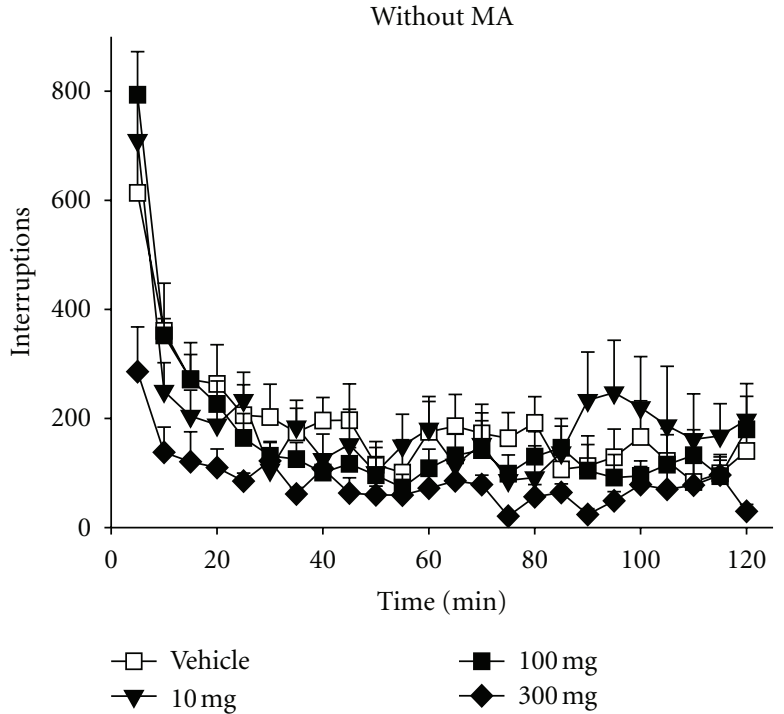

(b)

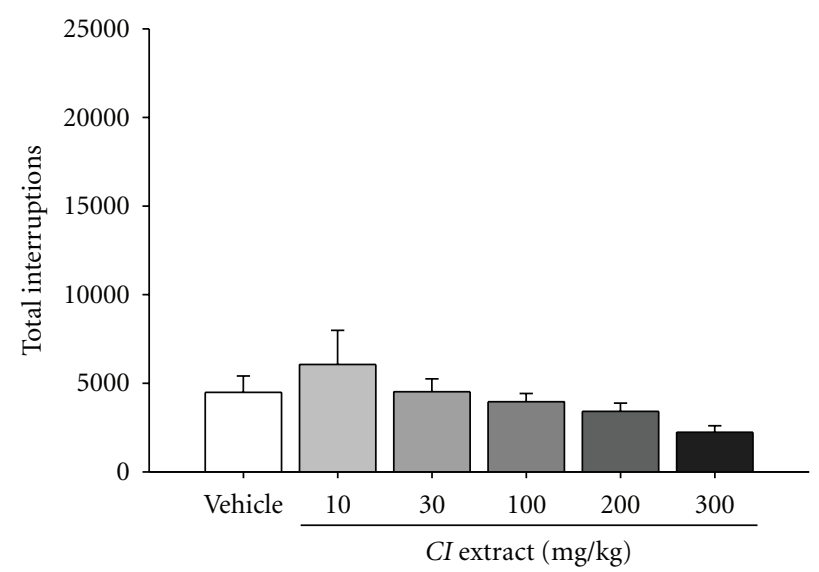

(d)

FIGURE 1: $\mathrm{Cl}$ extract dose-dependently inhibited hyperlocomotor activity induced by methamphetamine but not spontaneous locomotor activity. Effects of CI extract on the locomotor activity of mice with (a and c) or without (b and d) methamphetamine (MA) treatment. The locomotor activity was measured by horizontal and vertical interruptions of infrared (IR) beams in the testing chamber every 5 min for 2 hours after i.p. injection of $2 \mathrm{mg} / \mathrm{kg}$ methamphetamine (a) and (c) or normal saline (b) and (d). Mice were pretreated with CI extract (10-300 mg/kg, i.p.) or vehicle for $15 \mathrm{~min}$ before the test. (a) and (b): Time courses of changes of IR-beam interruptions after injection of methamphetamine (a) or normal saline (b) in groups pretreated with vehicle or various doses of CI extract. (a): For comparison, the vehicle group without MA pretreatment (open squares) was also included. Two-way ANOVA for MA-treated groups with repeated measures over time analysis indicated significant differences with a main effect of Treatment $\left(F_{3,621}=158.2 ; P<0.05\right)$ and Time $\left(F_{23,621}=15.63 ; P<0.05\right)$, and in the interaction of Treatment by Time $\left(F_{69,621}=0.34 ; P<0.05\right)$. (b): There was no significant difference among groups (two-way ANOVA). (c) and (d): Total interruptions in 2 hours after MA or saline treatment in various groups. ${ }^{* * *} P<0.001$ versus the vehicle group $\left(F_{5,48}=\right.$ 9.591; $P<0.001$ for (c); $F_{5,48}=2.045, P=0.086$ for $(\mathrm{d})$, one-way ANOVA with post hoc Tukey test). $N=9$.

the levels of control groups in both $71-115 \mathrm{~dB}$ and $77-$ $115 \mathrm{~dB}$ protocols (Figure 3(a)). Therefore, a PPI disruption model in mice was established by i.p. injection of $2 \mathrm{mg} / \mathrm{kg}$ methamphetamine, and this disruption can be prevented by typical and atypical antipsychotics.

\subsubsection{Extract Prevented Methamphetamine-Disrupted PPI.} Effects of CI extract (30 or $100 \mathrm{mg} / \mathrm{kg}$, i.p.) on methamphetamine-disrupted PPI were then examined. In mice pretreated with $C I$ extract for $15 \mathrm{~min}$, the magnitudes of PPI in response to $71-115 \mathrm{~dB}$ and $77-115 \mathrm{~dB}$ stimulations, respectively, were restored to $46.0 \pm 2.3 \%$ and $57.3 \pm 2.9 \%$ by $30 \mathrm{mg} / \mathrm{kg} \mathrm{CI}$ extract and to $53.2 \pm 3.4 \%$ and $67.2 \pm 2.5 \%$ by $100 \mathrm{mg} / \mathrm{kg}$ $C I$ extract, which were not significantly different from the control groups (Figure 3(b)).

In mice without treatment with methamphetamine, $C I$ Extract did not affect PPI (Figure 3(c)). Therefore, CI Extract is effective in protecting mice from the PPI disruption induced by methamphetamine at an optimal dose of $100 \mathrm{mg} /$ $\mathrm{kg}$ by i.p. injection, but not the PPI in normal mice. 


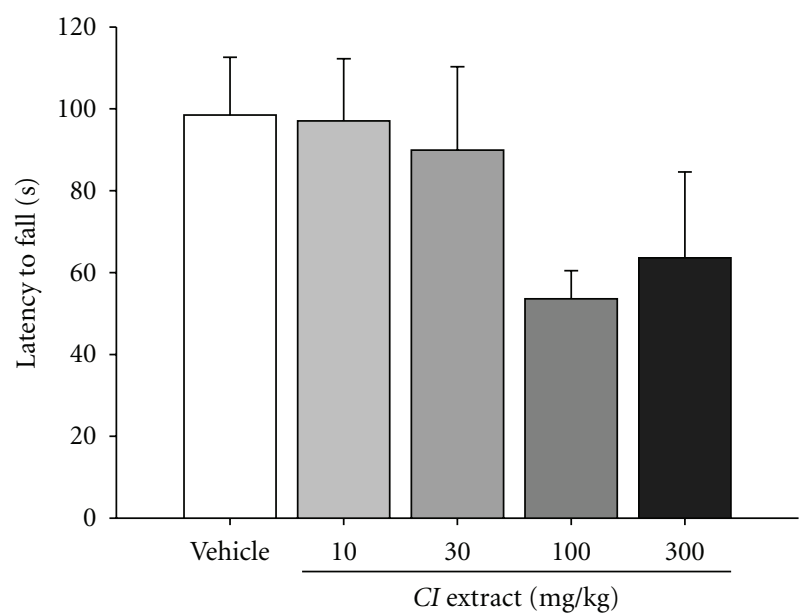

(a)

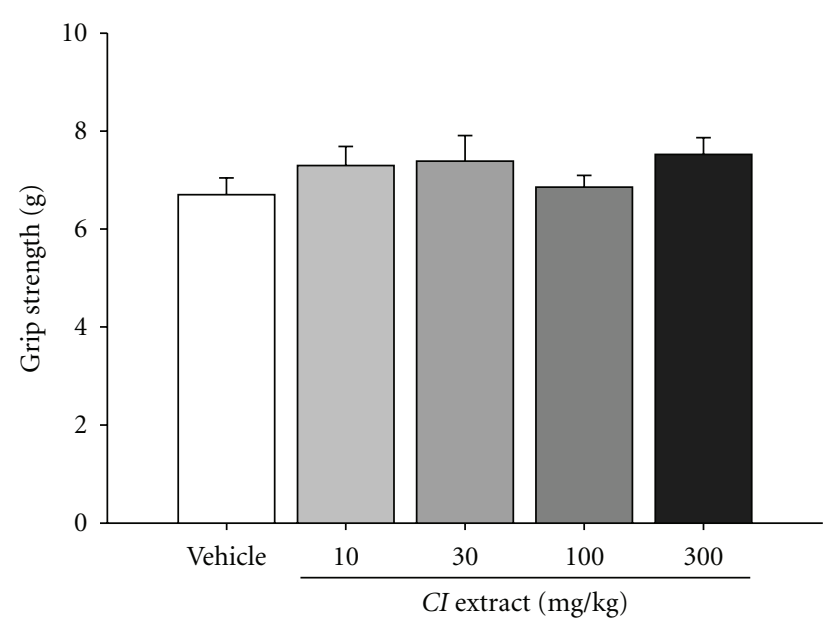

(b)

FIGURE 2: CI extract had no effect on motor coordination and muscle power. (a): Effects of CI extract on the motor coordination measured by the latency to fall in the rotarod test in mice. The mouse was pretrained until the latency to fall was greater than 120 sec. (b): Effects of CI extract on the grip strength (muscle power) of mice. The grip strength of forepaws of the mouse was measured by a grip strength meter three times every $2 \mathrm{~min}$ and averaged. Mice were pretreated with $C I$ extract and vehicle for $15 \mathrm{~min}$ before the tests. No significant difference was found among groups $\left(F_{4,40}=1.590, P=0.196\right.$ for $(\mathrm{a}) ; F_{4,40}=0.897, P=0.475$ for (b), one-way ANOVA). $N=9$.

3.2.3. CI Extract Prevented PPI Disruptions Induced by Ketamine. In addition to dopamine mimetic agents, PPI disruptions can also be induced by noncompetitive NMDA channel blockers, such as ketamine or MK-801 [10, 19, 22]. We, therefore, also examined if CI extract was effective in these PPI disruption models. In mice injected with ketamine $(30 \mathrm{mg} / \mathrm{kg}$, i.p.) for $10 \mathrm{~min}$, the magnitude of PPI in response to $71-115 \mathrm{~dB}$ stimulation was decreased from $58.9 \% \pm 2.3 \%$ to $43.0 \% \pm 2.2 \%$. Increasing the prepulse to $77 \mathrm{~dB}$, PPI was decreased from $65.6 \% \pm 3.1 \%$ to $52.3 \% \pm 2.8 \%$ (Figure $4(\mathrm{a})$ ). The PPI disruption induced by ketamine $(30 \mathrm{mg} / \mathrm{kg}$, i.p.) was not different from that induced by methamphetamine $(2 \mathrm{mg} / \mathrm{kg}$, i.p.) (Figure $4(\mathrm{a})$ versus Figure $3, P=0.662$ for $71-115 \mathrm{~dB}$ and $P=0.292$ for $77-115 \mathrm{~dB}$ protocols).

As in the PPI disruption model induced by methamphetamine, CI extract $(100 \mathrm{mg} / \mathrm{kg}$, i.p.) also prevented the PPI disruption induced by ketamine. The magnitudes of PPI in response to $71-115 \mathrm{~dB}$ and $77-115 \mathrm{~dB}$ stimulations were restored by $C I$ extract to $57.2 \% \pm 2.0 \%$ and $61.9 \% \pm$ $3.7 \%$, respectively, which were similar to control levels (Figure 4(a)).

3.2.4. CI Extract Prevented PPI Disruptions Induced by MK801. In mice receiving i.p. injection of $0.3 \mathrm{mg} / \mathrm{kg} \mathrm{MK-}$ 801 for $15 \mathrm{~min}$, the magnitude of PPI in response to a $71 \mathrm{~dB}$ prepulse acoustic stimulation was decreased from $58.9 \% \pm 2.3 \%$ to $41.5 \% \pm 2.9 \%$. Increasing the prepulse stimulus to $77 \mathrm{~dB}$, the PPI was decreased from $65.6 \% \pm$ $3.1 \%$ to $46.7 \% \pm 4.8 \%$ (Figure $4(\mathrm{~b})$ ). We, therefore, also successfully established a PPI disruption model induced by i.p. injection of MK-801. The PPI disruption induced by MK-801 (Figure 4(b)) was not significantly different from that by ketamine (Figure 4(a), $P=0.676,71-115 \mathrm{~dB}$ and $P=0.328,77-115 \mathrm{~dB}$ ) or by methamphetamine (Figure 3, $P=0.933,71-115 \mathrm{~dB}$ and $P=0.930,77-115 \mathrm{~dB})$. As in the PPI disruption model induced by methamphetamine or ketamine, CI extract $(100 \mathrm{mg} / \mathrm{kg}$, i.p.) also prevented the PPI disruption induced by MK-801 (Figure 4(b)). The magnitudes of PPI induced by $71-115 \mathrm{~dB}$ and $77-115 \mathrm{~dB}$ stimulations were restored by $C I$ extract to $53.8 \% \pm 1.3 \%$ and $58.9 \% \pm 0.9 \%$, respectively, which were similar to control levels.

3.2.5. Clinical Therapeutic Potential of CI. The patient in our case report [1] continues to visit our pediatric neurology clinic for her seizure control. On her latest visit to our clinic, being 6 years after she took CI, she reported that she had almost no more motor tics, which, when occurred occasionally, would subside 1 hour after taking CI. This argues against the possibility that her tic syndromes were naturally remitted with more matured brain circuits after growing up [4]. Given that antipsychotics were ineffective in this case, we suggest that the action mechanism(s) of $C I$ is other than directly blocking dopaminergic receptors. This inference is also supported by the effectiveness of $C I$ extract in rescuing the PPI disruptions induced by noncompetitive NMDA channel blockers (ketamine and MK801), for which dopamine receptor blockers are ineffective $[16,22]$. However, the possibility that $C I$ indirectly increases dopaminergic activity in the prefrontal cortex by increasing dopamine release or inhibiting dopamine degradation, such as catecholamine-O-methyl-transferase, cannot be excluded. Identification of the active constituent(s) in this ethanol extract of $C I$ leaves is undergoing in our laboratory and will be helpful in revealing the underline mechanism(s) of action of this herb. 


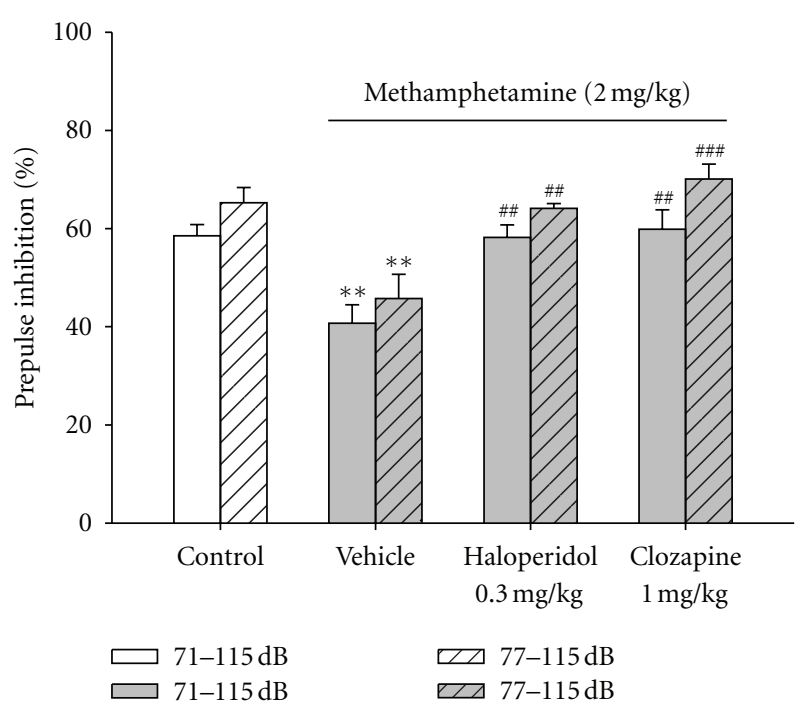

(a)

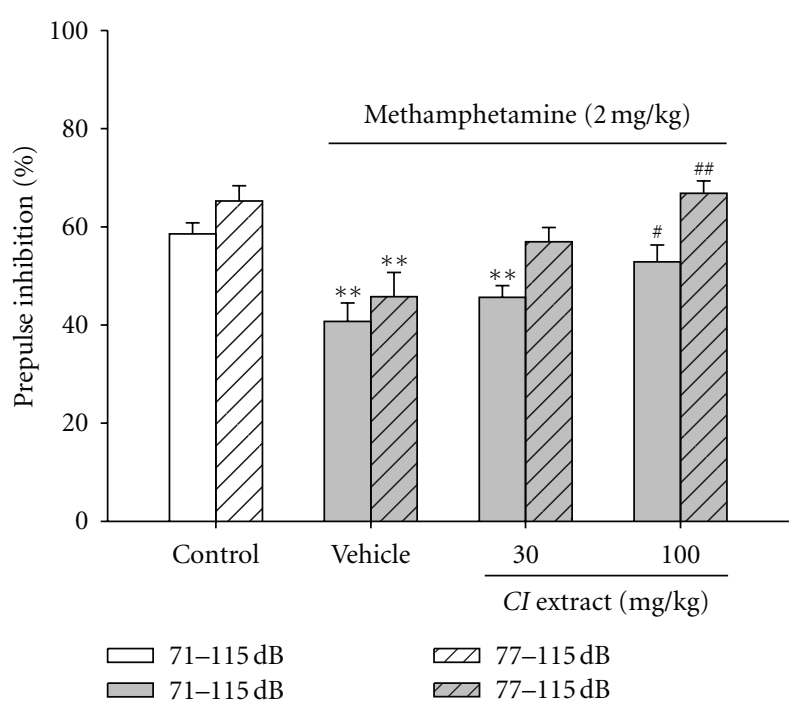

(b)

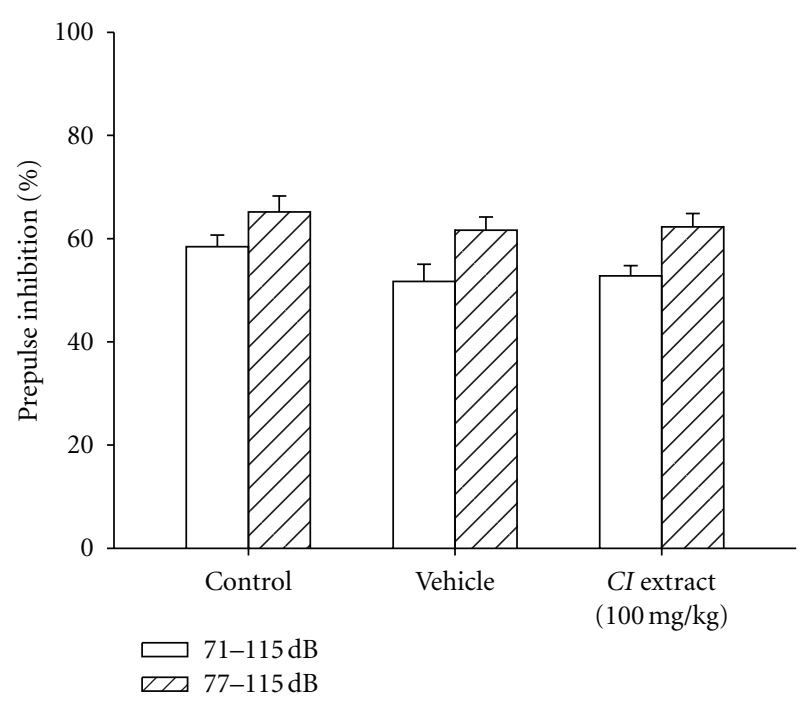

(c)

FIGURE 3: CI extract, like antipsychotics, prevented methamphetamine-induced disruption of prepulse inhibition of acoustic startle response (PPI) in mice. (a): Effects of haloperidol and clozapine, typical and atypical antipsychotic agents, respectively, on the PPI disruption induced by methamphetamine. (b): Effects of CI extract on the PPI disruption induced by methamphetamine. The magnitude of PPI in the startle response to a $115 \mathrm{~dB}$ acoustic stimulation paired with a prepulse of $71 \mathrm{~dB}(71-115 \mathrm{~dB})$ or $77 \mathrm{~dB}(77-115 \mathrm{~dB}) 120 \mathrm{~ms}$ ahead was measured as described in Section 2. Mice were pretreated with haloperidol ( $0.3 \mathrm{mg} / \mathrm{kg}$, i.p. ), clozapine ( $1 \mathrm{mg} / \mathrm{kg}$, i.p. $)$, CI extract (30 or $100 \mathrm{mg} / \mathrm{kg}$, i.p.), or vehicle (i.p.) for $15 \mathrm{~min}$, followed by methamphetamine ( $2 \mathrm{mg} / \mathrm{kg}$, i.p.) for $10 \mathrm{~min}$ before the test. (c): The effect of CI Extract on PPI in mice without methamphetamine treatment. Normal mice were treated with CI extract $(100 \mathrm{mg} / \mathrm{kg}$, i.p.) or vehicle (i.p.) for $15 \mathrm{~min}$ before being subjected to the PPI test. The control group is the mice treated with normal saline only (without methamphetamine). ${ }^{* *} P<0.01$ versus the

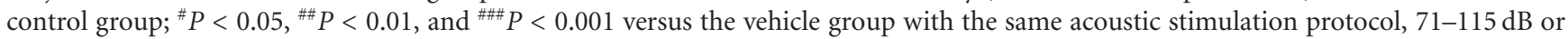
$77-115 \mathrm{~dB}$ (Student's $t$-test). $N=8$.

\section{Conclusions}

In summary, we found that $C I$ extract inhibited methamphetamine-induced hyperlocomotion and PPI disruptions induced by methamphetamine, ketamine, and MK801 at doses without affecting the spontaneous locomotor activity, rotarod performance, and grip force in mice. These findings suggest that $C I$ extract can relieve hyperlocomotion and improve sensorimotor gating deficit. The latter effect may prevent the urge of tic attacks in TS patients and be helpful for other psychiatric disorders such as schizophrenia, $\mathrm{ADHD}$, and OCD.

\section{Authors' Contribution}

H.-L. Chen, H.-J. Lee, and W.-J. Huang contributed equally to the paper. 


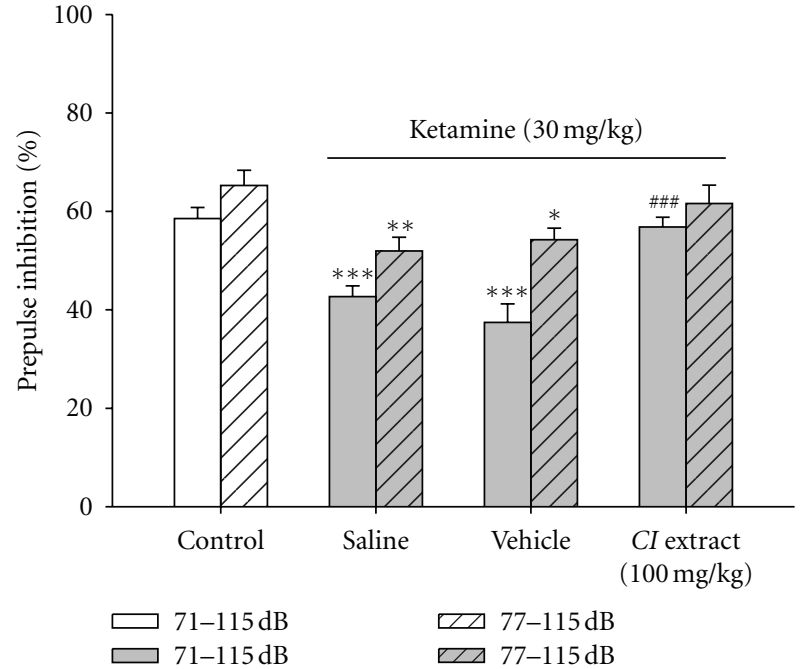

(a)

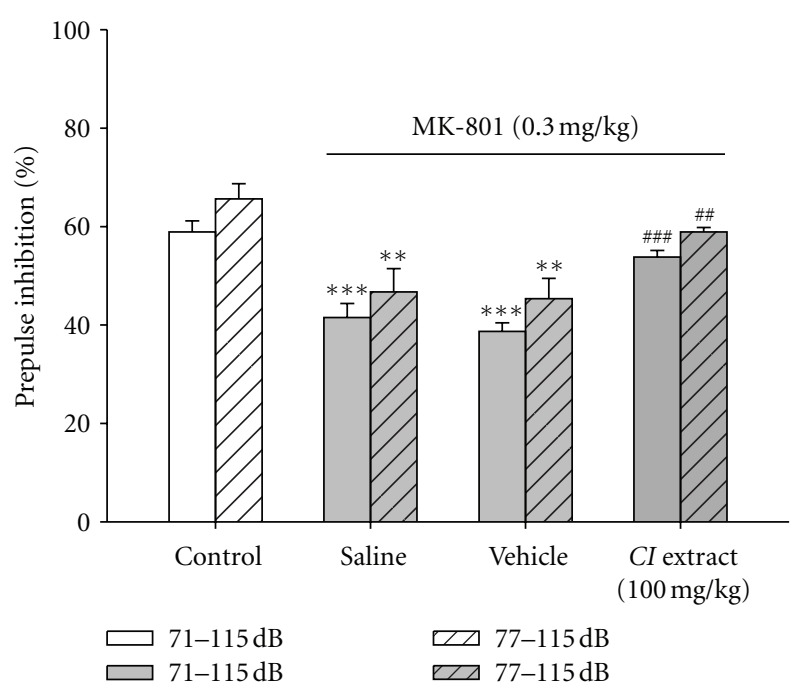

(b)

FIGURE 4: $C I$ extract prevented PPI disruptions induced by NMDA channel blockers in mice. Effects of $C I$ extract on PPI disruptions induced by NMDA channel blockers, ketamine (a) and MK-801(b). Mice were pretreated with $C I$ extract (100 mg/kg, i.p.) or vehicle for $15 \mathrm{~min}$, followed by ketamine (30 mg/kg, i.p.) for $10 \mathrm{~min}$ (a) or MK-801 (0.3 mg/kg, i.p.) for $20 \mathrm{~min}$ (b). The control group is the mice treated with normal saline alone (without NMDA blockers). The saline group is the mice pretreated with normal saline before being challenged with ketamine or MK-801. ${ }^{*} P<0.05,{ }^{* *} P<0.01$, and ${ }^{* * *} P<0.001$ versus the control group; ${ }^{\# \#} P<0.01$ and ${ }^{\# \# \#} P<0.001$ versus the vehicle group with the same acoustic stimulation protocol (Student's $t$ test). $N=8$.

\section{Acknowledgments}

This study was supported by the grants to L.-C. Chiou from National Science Council, Taipei, Taiwan (NSC 98-2323B002-012, NSC 99-2323-B002-012, NSC 100-2325-B002050, and NSC 98-2320-B-002-011-MY3), National Health Research Institutes, Miaoli, Taiwan (NHRI-EX99-9506NI), and National Taiwan University, Taipei, Taiwan (Excellent Research Grant 99R81855) and the grant to W.-J. Huang from National Science Council, Taipei, Taiwan (NSC 1002320-B-038-006). The authors thank the support from Behavior Core, Neurobiology and Cognitive Center, National Taiwan University.

\section{References}

[1] P. C. Fan, W. J. Huang, and L. C. Chiou, "Intractable chronic motor tics dramatically respond to Clerodendrum inerme (L) gaertn," Journal of Child Neurology, vol. 24, no. 7, pp. 887-890, 2009.

[2] M. M. Robertson, "Diagnosing Tourette syndrome is it a common disorder?" Journal of Psychosomatic Research, vol. 55, no. 1, pp. 3-6, 2003.

[3] H. S. Wang and M. F. Kuo, “Tourette's syndrome in Taiwan: an epidemiological study of tic disorders in an elementary school at Taipei County," Brain and Development, vol. 25, supplement 1, pp. S29-S31, 2003.

[4] H. S. Singer, "Tourette's syndrome: from behaviour to biology," The Lancet Neurology, vol. 4, no. 3, pp. 149-159, 2005.

[5] R. Kurlan, D. Whitmore, C. Irvine, M. P. McDermott, and P. G. Como, "Tourette's syndrome in a special education population: a pilot study involving a single school district," Neurology, vol. 44, no. 4, pp. 699-702, 1994.
[6] H. S. Singer, "Discussing outcome in tourette syndrome," Archives of Pediatrics and Adolescent Medicine, vol. 160, no. 1, pp. 103-105, 2006.

[7] H. S. Singer and K. Minzer, "Neurobiology of Tourette's syndrome: concepts of neuroanatomic localization and neurochemical abnormalities," Brain and Development, vol. 25, supplement 1, pp. S70-S84, 2003.

[8] J. F. Leckman, D. E. Walker, and D. J. Cohen, "Premonitory urges in Tourette's syndrome," American Journal of Psychiatry, vol. 150, no. 1, pp. 98-102, 1993.

[9] N. R. Swerdlow, B. Karban, Y. Ploum, R. Sharp, M. A. Geyer, and A. Eastvold, "Tactile prepuff inhibition of startle in children with Tourette's syndrome: in search of an "fMRIfriendly" startle paradigm," Biological Psychiatry, vol. 50, no. 8, pp. 578-585, 2001.

[10] N. R. Swerdlow, M. A. Geyer, and D. L. Braff, "Neural circuit regulation of prepulse inhibition of startle in the rat: current knowledge and future challenges," Psychopharmacology, vol. 156, no. 2-3, pp. 194-215, 2001.

[11] F. X. Castellanos, E. J. Fine, D. Kaysen, W. L. Marsh, J. L. Rapoport, and M. Hallett, "Sensorimotor gating in boys with Tourette's syndrome and ADHD: preliminary results," Biological Psychiatry, vol. 39, no. 1, pp. 33-41, 1996.

[12] E. M. Ornitz, G. L. Hanna, and J. de Traversay, "Prestimulation-induced startle modulation in attention-deficit hyperactivity disorder and nocturnal enuresis," Psychophysiology, vol. 29, no. 4, pp. 437-451, 1992.

[13] K. Hoenig, A. Hochrein, B. B. Quednow, W. Maier, and M. Wagner, "Impaired prepulse inhibition of acoustic startle in obsessive-compulsive disorder," Biological Psychiatry, vol. 57, no. 10, pp. 1153-1158, 2005.

[14] D. Braff, C. Stone, E. Callaway, M. Geyer, I. Glick, and L. Bali, "Prestimulus effects on human startle reflex in normals and 
schizophrenics," Psychophysiology, vol. 15, no. 4, pp. 339-343, 1978.

[15] J. Kerbeshian, C. Z. Peng, and L. Burd, "Tourette syndrome and comorbid early-onset schizophrenia," Journal of Psychosomatic Research, vol. 67, no. 6, pp. 515-523, 2009.

[16] M. A. Geyer and B. Moghaddam, "Animal models relevant to schizophrenia disorders," in Neuropsychopharmacology: The Fifth Generation of Progress, K. L. Davis, D. Charney, J. T. Coyle, and C. Nemeroff, Eds., pp. 689-701, American College of Neuropsychopharmacology, 2002.

[17] D. C. Javitt and S. R. Zukin, "Recent advances in the phencyclidine model of schizophrenia," American Journal of Psychiatry, vol. 148, no. 10, pp. 1301-1308, 1991.

[18] R. S. Mansbach, M. A. Geyer, and D. L. Braff, "Dopaminergic stimulation disrupts sensorimotor gating in the rat," Psychopharmacology, vol. 94, no. 4, pp. 507-514, 1988.

[19] R. S. Mansbach and M. A. Geyer, "Effects of phencyclidine and phencyclidine biologs on sensorimotor gating in the rat," Neuropsychopharmacology, vol. 2, no. 4, pp. 299-308, 1989.

[20] R. S. Mansbach and M. A. Geyer, "Parametric determinants in pre-stimulus modification of acoustic startle: interaction with ketamine," Psychopharmacology, vol. 105, no. 2, pp. 162-168, 1991.

[21] N. R. Swerdlow, D. L. Braff, M. A. Geyer, and G. F. Koob, “Central dopamine hyperactivity in rats mimics abnormal acoustic startle response in schizophrenics," Biological Psychiatry, vol. 21, no. 1, pp. 23-33, 1986.

[22] M. A. Geyer, K. Krebs-Thomson, D. L. Braff, and N. R. Swerdlow, "Pharmacological studies of prepulse inhibition models of sensorimotor gating deficits in schizophrenia: a decade in review," Psychopharmacology, vol. 156, no. 2-3, pp. 117-154, 2001. 


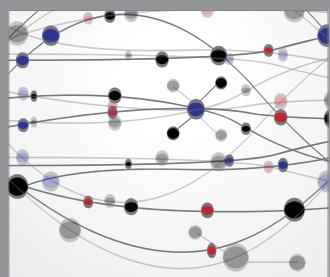

The Scientific World Journal
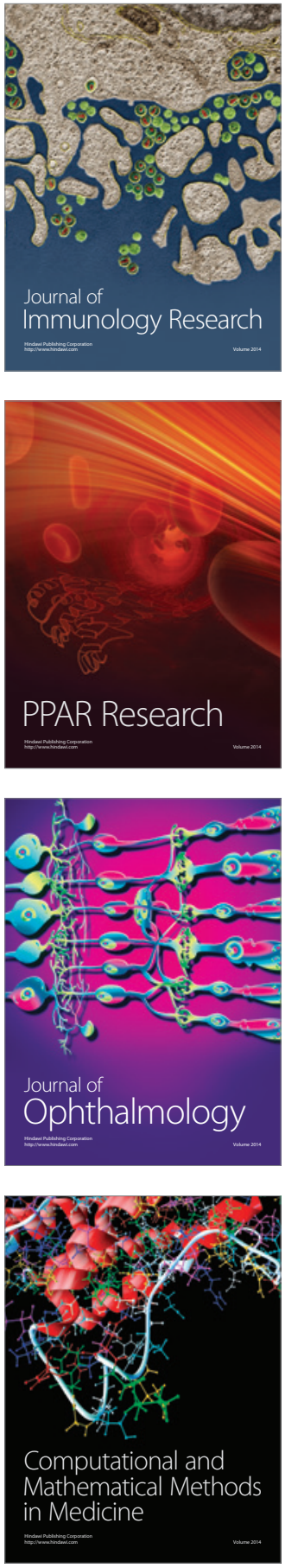

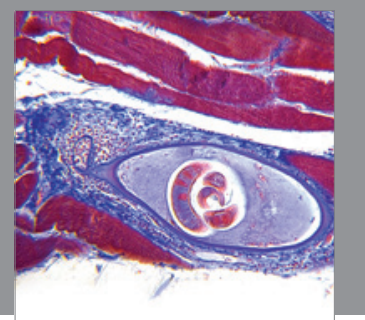

Gastroenterology

Research and Practice
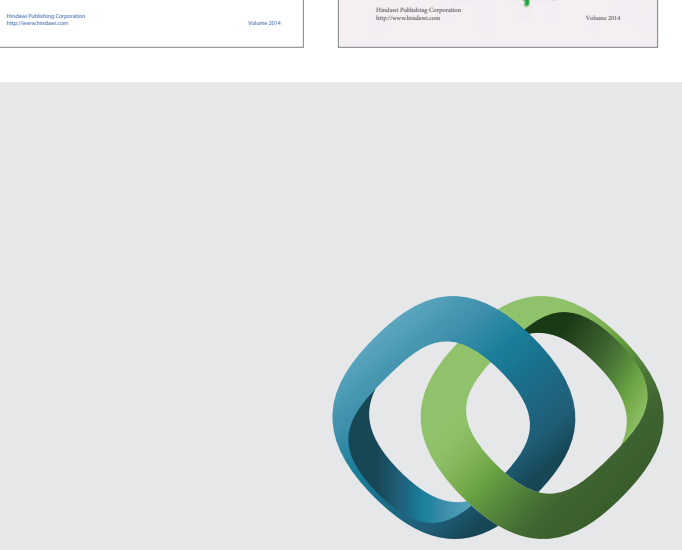

\section{Hindawi}

Submit your manuscripts at

http://www.hindawi.com
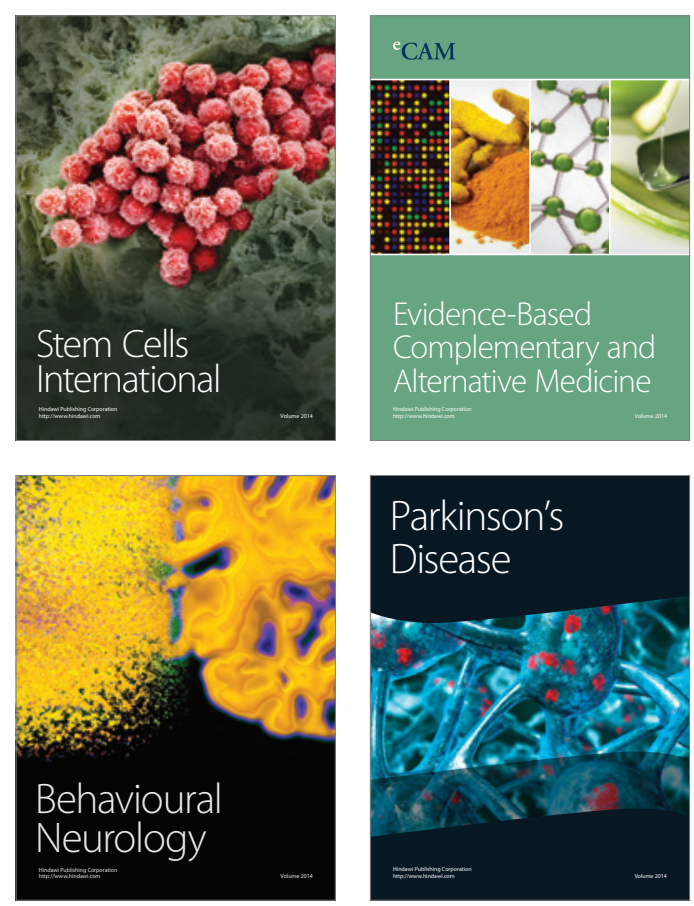

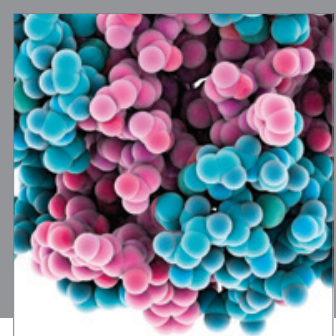

Journal of
Diabetes Research

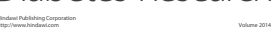

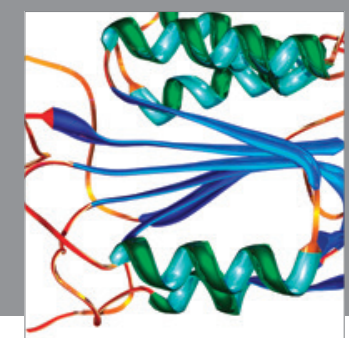

Disease Markers
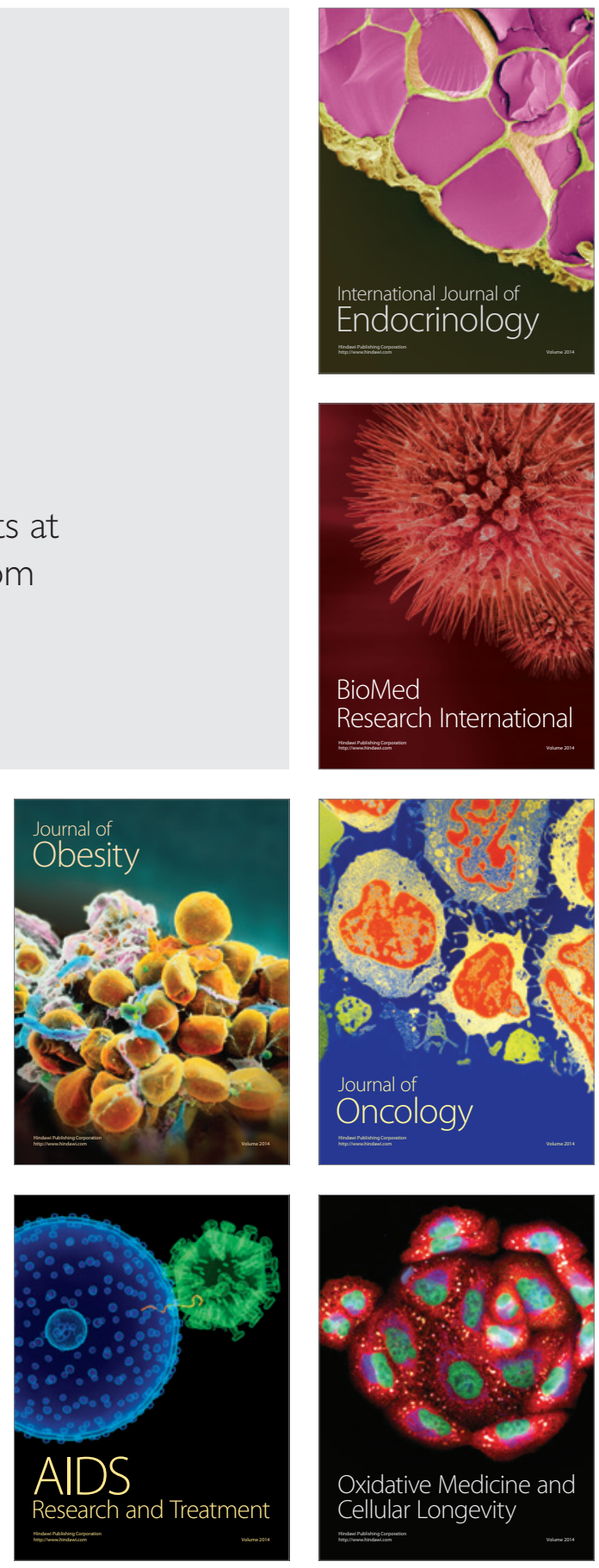